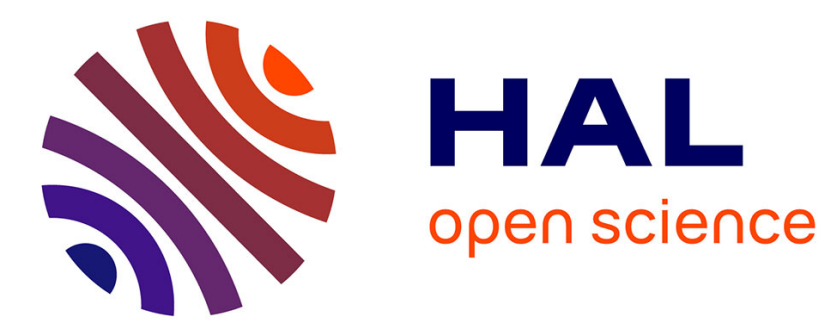

\title{
Éducation et musée
}

Marie-Sylvie Poli

\section{To cite this version:}

Marie-Sylvie Poli. Éducation et musée. Culture et Musées, 2021, La muséologie : 20 ans de recherches, Hors-série, pp.165-187. 10.4000/culturemusees.757 . hal-03271207

\section{HAL Id: hal-03271207 https://hal.science/hal-03271207}

Submitted on 25 Jun 2021

HAL is a multi-disciplinary open access archive for the deposit and dissemination of scientific research documents, whether they are published or not. The documents may come from teaching and research institutions in France or abroad, or from public or private research centers.
L'archive ouverte pluridisciplinaire HAL, est destinée au dépôt et à la diffusion de documents scientifiques de niveau recherche, publiés ou non, émanant des établissements d'enseignement et de recherche français ou étrangers, des laboratoires publics ou privés. 


\section{CpenEdition Journals \\ Culture \& Musées}

Muséologie et recherches sur la culture

Hors-série | 2013

La muséologie : 20 ans de recherches

\section{Éducation et musée}

Education and the Museum

Educación y museo

\section{Marie-Sylvie Poli}

p. $165-187$

https://doi.org/10.4000/culturemusees.757

\section{Résumés}

Français English Español

Les récentes études de publics le confirment, on va au musée en 2012 avec la même envie d'éducation qu'on y allait à la fin des années quatre-vingt-dix : pour apprendre, se cultiver, se former et développer son esprit critique. En relation avec cette continuité dans les représentations sociales, les recherches sur « éducation et musée » ont progressé durant cette même période, passant d'une approche pédagogique et didactique à une perspective communicationnelle et pragmatique. Nous présentons dans cet article une synthèse des problématiques et des notions qui depuis vingt ans caractérisent ce champ de la recherche en France. Nous insistons en particulier sur les apports conjoints des professionnels des musées, des pédagogues et des universitaires aux recherches sur l'éducation muséale. On voit que l'arrivée du numérique de la connaissance au musée offre de stimulantes perspectives de recherche sur culture numérique, éducation et musée.

Recent visitor research findings have shown that people go nowadays to museums with the same desire for education as they went in the nineties : to learn, to become well-educated and to develop their critical faculties. In connection with this continuity in social representation, research on « education and the museum » has progressed during the same period, changing from a pedagogical and didactic approach to a focus on communication and the pragmatic perspective. In this article we shall present an overview of the questions and concepts that have characterized this area of research in France over the last twenty years. We shall highlight, in particular, the contributions on museum education produced jointly by museum professionals, educators and university research teams. We show that the development of digital knowledge management leads to very stimulating perspectives in the research into digital culture, education and the museum.

Los recientes estudios de públicos lo confirman, vamos al museo en 2012 con las misma disposición de educación con que íbamos a fines de los noventa : para aprender, educarse, formarse y desarrollar el espíritu crítico. En relación a esta continuidad en las representaciones sociales, las investigaciones sobre « educación y museo » han evolucionado durante este mismo periodo, pasando de un enfoque pedagógico y didáctico a una perspectiva comunicacional y pragmática. Presentamos en este artículo 
una síntesis de las problemáticas y de las nociones que hace veinte años caracterizan este campo de investigación en Francia. Insistimos en particular sobre los aportes conjuntos de los profesionales de los museos, de los pedagogos y de los universitarios en las investigaciones sobre la educación museística. Observamos que la llegada de lo digital del conocimiento en el museo ofrece estimulantes perspectivas de investigación sobre cultura digital, educación y museo.

\section{Entrées d'index}

Mots-clés : musée, éducation, pédagogie, didactique, école, culture numérique Keywords: museum, education, pedagogy, didactics, school, digital humanities Palabras clave: museo, educación, pedagogía, didáctica, escuela, cultura digital

\section{Texte intégral}

Musée et éducation seraient-ils indéfectiblement unis au point de pouvoir être envisagés comme un paradigme culturel ?

2 C'est la position que semble défendre le Conseil international des musées (ICOM) dans la définition qu'il donne en 2007 du musée, dès le troisième article de ses statuts (article 3 : Définition des termes. Section $1:$ Musée) : "Le musée est une institution permanente sans but lucratif, au service de la société et de son développement, ouverte au public, qui acquiert, conserve, étudie, expose et transmet le patrimoine matériel et immatériel de l'humanité et de son environnement à des fins d'études, d'éducation et de délectation. » (Statuts de I'ıcom, art. 3 § 1.)

3 Il est opportun, pour introduire ce regard porté ici sur la recherche en France à propos d'éducation et musée durant les vingt dernières années, de rappeler cette définition de I'ıсом. Car, à l'aune de la dimension internationale de ses réseaux de professionnels et de chercheurs, en préférant le terme « éducation » à celui d' " instruction » au titre d'une des trois missions centrales du musée (encadrée par les missions d'études et de délectation), I'ıсом précise que l'aspect éducatif du musée est une dimension culturelle et interculturelle d'échange et de communication allant bien au-delà du pédagogique stricto sensu.

4 Il ne sera pas question dans cet article de définir les concepts d'éducation et d'instruction en les replaçant respectivement dans le champ muséal mais plutôt de saisir pourquoi, en muséologie, il est essentiel de s'en tenir aux distinctions sémiques que la langue française établit entre ces deux substantifs : « L'instruction est relative à l'esprit et s'entend des connaissances que l'on acquiert et par lesquelles on devient habile et savant. L'éducation est relative à la fois au cœur et à l'esprit, et s'entend des connaissances que l'on fait acquérir et des directions morales que l'on donne aux sentiments. " (Littré, version électronique.)

5 La première distinction tient à la forme conjuguée active versus forme infinitive passive attribuée au verbe acquérir ainsi qu'au rôle d'actant attribué au sujet grammatical dans l'emploi de ce verbe ; dans l'instruction, on acquiert des connaissances (l'apprenant est actif), alors que l'éducation s'entend des connaissances que l'on fait acquérir (c'est le médiateur qui agit).

6 Ainsi, d'un point de vue sémantique, l'éducation relèverait de la médiation reposant sur une prise en charge du sujet par un tiers, alors que l'instruction renverrait au champ de la construction de soi par une démarche volontariste personnelle du sujet.

7 L'opérateur qui fait acquérir les connaissances s'avère donc central dans l'éducation, qu'il s'agisse d'un opérateur institutionnel (école, musée, entreprise, bibliothèque, etc.), d'un opérateur humain (enseignant, médiateur, formateur, parent, etc.) ou d'un opérateur technique (livre, média, outil numérique, etc.).

8 La seconde distinction tient aux champs lexicaux sollicités dans les définitions des deux termes : le champ lexical de l'esprit, du savoir et des compétences pour l'instruction - un champ lexical également présent dans la définition d'éducation, mais auquel on ajoute 
celui des sentiments, du cœur et de la morale. C'est-à-dire les valeurs symboliques qui distinguent la culture du savoir et la médiation du seul transfert d'information (Topalian \& Le Marec, 2003).

9 C'est cette conception moderne de l'éducation, communicationnelle autant que didactique, où apprendre donne lieu à des changements d'attitudes et de croyances, que nous avons discernée dans les travaux des vingt dernières années auxquels la présente synthèse fait référence. La visite au musée y rimera donc autant avec symbolique et social qu'avec informatif et cognitif (Dierking, 1994).

10 Cette conception culturelle de l'éducation au et par le musée, dans laquelle le visiteur quel qu'il soit est considéré comme curieux, sensible et intelligent, constitue le fil directeur du regard que nous avons porté sur le thème éducation et musée dans la recherche en muséologie en France depuis les années quatre-vingt-dix. Ces recherches, on le verra, permettent de penser le musée non pas comme une boîte à contenus, un écrin pour objets et modes de vie patrimonialisés, mais comme un miroir en perpétuel changement de savoirs et de valeurs constamment modifiés et renégociés par chaque société.

En cela, nous situerons notre quête dans un cadre épistémologique qui, sans pour autant coller parfaitement à la définition du musée qu'en donne l'ıcom, va nous conduire à concevoir le concept de musée à partir d'une problématique plus culturelle que pédagogique (Desvallées \& Mairesse, 2005). Même si, on le verra, les recherches en didactique sont celles qui envisagent tout particulièrement le musée comme un outil d'instruction au sens technique du terme.

La variété des thèmes et des cadres théoriques des recherches menées dans le domaine " éducation et musée » depuis les années quatre-vingt-dix est riche de la diversité des points de vue et des sensibilités des chercheurs formés à des écoles théoriques diverses. Nous en présentons ici une vision inévitablement synthétique, organisée en deux parties : 1) les problématiques qui structurent le champ sur cette période ; 2) les notions opératoires qui caractérisent, du point de vue lexical, le discours des recherches sur " éducation et musée ", notions dont l'hétérogénéité énonciative hypothèque la convergence des problématiques autour d'un cadre théorique commun.

Les recherches à dimension programmatique dans lesquelles le numérique (ou le virtuel) est envisagé comme une innovation sociale qui bouscule profondément nos modes de relations à la culture, au savoir, donc à l'éducation et au musée, interpellent depuis quelques années déjà les chercheurs français. Elles augurent de nouvelles recherches, prometteuses et à dimension internationale. Apparues depuis peu, malgré tout l'intérêt que nous leur portons, elles ne seront évoquées qu'à la fin de l'article.

\section{Les problématiques}

Une partie des recherches qui ont attiré notre attention pour cette rétrospective sur " éducation et musée » a pour ambition d'établir des cadres théoriques et des problématiques ad hoc dans le but qu'ils s'avèrent pertinents pour tous les chercheurs qui engagent des travaux dans ce champ. Le numéro 7 de la revue Publics \& Musées est exemplaire de cette focalisation de la démarche épistémologique (1995).

\section{Des problématiques partagées par des chercheurs et des professionnels du musée}

On constate globalement que les chercheurs français prolongent la démarche engagée par les chercheurs canadiens qui, dès les années quatre-vingt-dix, ont produit des publications thématiques pour établir l'état de l'art sur la question de l'éducation muséale (Dufresne-Tassé, 1991). 
Le champ se caractérise par le fait que les auteurs ne sont pas uniquement des universitaires ou des chercheurs institutionnels. Le métissage actoriel dans les projets de recherche, qui se traduit par des cosignatures des publications, témoigne de la capacité d’innovation et de réflexivité critique des acteurs du domaine.

On notera aussi que les auteurs revendiquent explicitement leur statut de professionnels des musées, de pédagogues, de formateurs ou d'experts en médiation (Eidelman, Roustan \& Goldstein, 2007).

18 Ainsi, certains auteurs, engagés dans des institutions muséales en tant qu'enseignant détaché, responsable des publics scolaires ou chercheur en sciences de l'éducation en poste dans un musée, mènent des recherches à caractère fréquemment prosélyte en faveur du musée, des partenariats école-musée (Fortin-Debart, 2004) ou encore de l'ouverture du musée aux publics dits éloignés ou empêchés (Chauvey, 2010 ; Julien, 2004). Et ces recherches sont sous-tendues par la constance, dès leur thèse, d'une ou plusieurs problématiques auxquelles on peut assez facilement associer ces auteurs durant une bonne partie au moins de leur carrière.

$\mathrm{Au}$ croisement de ces problématiques, on observe un large consensus dans les publications des vingt dernières années sur le fait que le musée est une institution à vocation éducative au sens de «faire ressentir et comprendre » plutôt qu'au sens de «faire apprendre et vulgariser » (Chaumier, 2008 ; Le Marec, 2006).

20 A contrario, on trouve un petit nombre de travaux critiques vis-à-vis du concept de « musée dispositif éducatif » (Schiele \& Boucher, 2001 ; Ruddel, 2005). Lorsque c'est le cas, la médiation muséale y est analysée comme étant certes capable de porter les questions scientifiques à la connaissance du grand public, mais elle est également accusée de faire écran à la capacité critique du visiteur.

21 La muséologie étant une discipline dont les recherches appliquées nourrissent la recherche théorique, la littérature sur " éducation et musée » est elle aussi riche de travaux d'analyse pragmatique d'expositions menés par des spécialistes en médiation (Chaléon, Demarq \& Maggioni, 2010) et par des chercheurs en sémiotique experts dans l'analyse de dispositifs (Flon, 2006), d'opérations menées auprès de publics cibles (Girault (dir.), 2003) ou d'évaluations de médiations des savoirs (Poli, 2008).

\section{Types de publics et terrains : des problématiques spécifiques}

Si dans les années quarante à quatre-vingt la majorité de la recherche consacrée à « éducation et musée » était réalisée à partir d'études de cas menées dans des musées de sciences, des centres d'interprétation ou des aquariums (Maigret, 2005), les musées de société et les musées d'histoire mais également les musées d'art sont désormais pris comme terrains d'analyse (Gottesdiener, 1992 ; Kindler \& Darras, 1998).

23 Les expérimentations pédagogiques menées dans le cadre de contrats école-musée participent à enrichir un domaine de recherche plutôt interdisciplinaire, au centre duquel on trouve les sciences de la vie et de la terre, la chimie, l'histoire, mais également les lettres ou l'histoire de l'art (Triquet \& Poli, 2001). Dès lors qu'ils analysent tout dispositif à l'aune de sa visée éducative, les chercheurs considèrent l'exposition comme un dispositif éducatif légitimé par le musée.

24 Les publics sur lesquels portent les recherches sont extrêmement hétérogènes. Certains se centrent sur des publics particuliers : les enfants, les jeunes, les familles, les malvoyants, les publics éloignés, etc. Ces recherches sur ces différentes catégories de visiteurs et d'apprenants peuvent se lire comme l'effet d'expérimentations tentées par des professionnels du musée pour toucher le mieux possible et de la façon la plus pertinente des personnes de tout milieu, de toute condition, de toute origine et de tout âge.

25 Parfois la recherche de terrain sur l'éducation rejoint les préoccupations de rentabilité de plus en plus imposées aux musées au titre de l'investissement productif. Le débat porte 
alors sur l'idée que le sens de la communauté, la mission d'éducation et le souci financier ne devraient pas être envisagés comme irrémédiablement incompatibles (Mairesse, 2000).

On notera que, dans l'ensemble, les auteurs qui signent ou cosignent les travaux sur ces problématiques sont avant tout des chercheurs universitaires, des docteurs en muséologie et des doctorants inscrits dans des projets de thèse menés sur des terrains muséaux. Ces sujets étant historiquement interdisciplinaires, les auteurs peuvent être rattachés aux sciences de l'éducation, aux sciences de l'information et de la communication, à la didactique des sciences, à la sociolinguistique, à la sociologie également. Ces thèmes favorisent parfois également les cosignatures entre un chercheur et un professionnel de musée ou un médiateur, entre des éducateurs et des muséologues (Mariani-Ducray \& Wasserman, 2007).

\section{Des problématiques variées et novatrices}

Ces dix dernières années, les travaux se caractérisent par des questions posées à des objets de recherche de plus en plus précis, de plus en plus spécialisés. Ainsi, les catégories de visiteurs qui font l'objet d'observations et d'entretiens sont-elles de plus en plus étroites, comme les familles, les enfants de quatre à six ans, par exemple (Cordier \& Serre, 2000), les adolescents ou les familles en difficulté (Ailincai, 2008). Au fil des publications et des recherches, on voit les objets et les terrains d'études se spécialiser en considérant les types de classes ou d'établissements, les variables socio-économiques des quartiers concernés par les « recherches action » (Jonchery, 2005), les langues pratiquées par les visiteurs et les apprenants (Rigat, 2005), les types d'aides à la visite, les technologies de médiation, les motivations personnelles à la visite, etc.

28 Des recherches sont menées sur les ateliers pédagogiques, sur les classes au musée (Porcedda, 2006), sur les comités d'entreprise au musée, sur les cartels interactifs et autres outils à vocation pédagogique. Là encore, l'évolution des terrains et des objets de recherche durant vingt ans permet une approche réflexive sur le sujet " éducation et musée ». Car si les recherches engagées le sont désormais sur des dispositifs muséaux qui n'existaient pas il y a peu, la réciprocité est également à prendre en compte. La capacité des musées à innover dans leur conception de l'éducation et dans leurs représentations des attentes des visiteurs permet ainsi aux chercheurs de formaliser de nouveaux objets de recherche, en lien direct avec les attentes sociales (Cohen-Azria, 2002b).

En marge de ces recherches à dimension sociologique, on constate que le patrimoine scolaire et éducatif au musée intéresse nombre de chercheurs centrés sur les questions des politiques d'enrichissement, de gestion et de valorisation des collections pédagogiques. C'est un domaine de recherche qui continue de se développer activement (Boyer, 2009).

\section{La problématique traditionnelle des rapports entre l'école et le musée}

30 Les travaux de recherche qui portent sur les rapports entre l'école et le musée constituent le corpus quantitativement le plus étoffé de la littérature sur " éducation et musée ». En effet, dès lors qu'un chercheur décide de travailler sur ce thème, il est rare qu'il ne fasse pas un détour par les relations qui, depuis les années trente, ont rapproché l'univers du musée du monde de l'institution scolaire.

31 Cadrages historiques, comparaisons entre les musées de sciences et les autres types de musée, création d'un statut spécifique pour les enseignants détachés, analyse critique des dispositifs d'évaluation des savoirs acquis lors de la sortie au musée, mais aussi questionnement sur le statut de l'élève au musée, ces travaux se caractérisent généralement par une présentation prosélyte de la visite de la classe au musée et par un discours critique souvent assez virulent à propos du déficit présumé de communication entre ces deux 
institutions emblématiques de la conception laïque et hégémonique du savoir et de la culture (Cohen-Azria, 2000).

Cette problématique, on l'a déjà fait remarquer, ouvre la voie à des recherches interdisciplinaires (didactique et muséologie, psychopédagogie et muséologie, didactique et sémiologie, didactique et communication) dans lesquelles les préoccupations des pédagogues croisent celles des professionnels des musées sur des questions aussi variées que l'écriture des outils de médiation (Poli, 2002), les programmes de formation des enseignants ou la question de l'apprentissage par le jeu et l'émotion (Profit, 2009).

\section{Les relations entre musée et éducation au sens large}

Les recherches traitant de l'éducation populaire et du musée, de la nouvelle muséologie et de la muséologie collaborative relèvent peu ou prou des travaux produits par des chercheurs qui s'intéressent aux relations entre musée et éducation au sens large (Maurel, 2009). La question de l'éducation est tellement consubstantielle de la question de la médiation muséale qu'il est quasiment impossible de tracer une ligne de partage entre les recherches en muséologie et celles sur le musée comme dispositif d'éducation. Sur les vingt dernières années qui nous occupent ici, la question de la transmission des connaissances et celle de la transmission de valeurs lors de la visite de musée ou d'exposition sont inextricablement liées et ce, de plus en plus. Les recherches les plus récentes sur la réception par les visiteurs renforcent le postulat selon lequel il n'existe de savoirs qu'inscrits dans un discours, lui-même toujours nourri de valeurs et de subjectivité ; et cela, qu'il s'agisse du musée de sciences, du musée d'histoire, du musée de société ou du musée d'art (Poli \& Idjeraoui-Ravez, 2011). Il est ainsi désormais acquis que les musées exposent autant des savoirs que des points de vue de commissaires d'expositions ou de spécialistes par l'intermédiaire d'écritures muséographiques par nature subjectives (Meunier, Romano \& Jacobi, 2000). La question de la transmission de la connaissance au/par le musée ne se limite donc plus à une question de captation de l'intelligence par le discours objectif scientifique ; le recours à des procédés scénographiques ou expographiques qui visent à toucher le visiteur par l'émotion est désormais acté par les pédagogues, les formateurs et par les médiateurs (Molinati \& Girault, 2007). Les études de publics les plus récentes démontrent, qui plus est, que les visiteurs déclarent plus échanger avec leurs proches à propos des émotions ressenties en se rendant au musée que des connaissances qu'ils ont le sentiment d'avoir acquises. Il n'empêche, les visiteurs revendiquent toujours leur volonté et leur goût d'apprendre lorsqu'ils vont au musée, seuls ou accompagnés (Eidelman \& Jonchery, 2011).

\section{La problématique de l'éducation non formelle au musée}

Certaines recherches interdisciplinaires prennent pour objet d'analyse le possible rapprochement entre éducation muséale et éducation non formelle. Relève de l'éducation non formelle toute la palette des actions de formation (dont la formation permanente) et d'éducation (dont l'éducation populaire) qui, d'une part est réalisée à partir de la seule volonté de celui qui s'y adonne, et d'autre part n'est ni étalonnée par des niveaux de connaissances propres au système éducatif ni sanctionnée par des évaluations notées débouchant sur l'obtention de diplômes (Colardelle, 2007).

L'éducation muséale, quant à elle, est réalisée par le biais de formations organisées par des professionnels de musée. Celles-ci sont destinées à des enseignants ou à des scolaires. Il s'agit de formations non évaluées, centrées sur la découverte des métiers du musée ainsi 
que sur les différents modes de fonctionnement de l'institution (gestion des collections, montage d'exposition, conception d'un projet muséographique, etc.). Dans cette approche restrictive, la formation muséale consiste à apprendre comment vit le musée, quels sont ses enjeux, qui sont les professionnels qui le font fonctionner, et comment ces derniers travaillent à concevoir des dispositifs de communication pour accueillir les visiteurs. Les chercheurs travaillant sur ces questions déplacent leurs analyses des contenus de savoirs disciplinaires vers l'intérêt que comporte pour un citoyen la connaissance des coulisses et des enjeux politiques du musée en tant que lieu de rencontres et d'échanges pour des personnes venues de tous horizons (Meunier, 2008).

\section{Le musée et/ou l'exposition envisagés comme des médias éducatifs}

36 Les recherches en sciences de l'information et de la communication des années quatrevingt-dix se caractérisent en France par un courant de travaux sur les médias, engagés par des chercheurs formés à la sémiologie ou à la sémiotique. Le propre de ces recherches est de systématiquement réintroduire dans leurs analyses la question du social et de l'opérativité symbolique des différents médias, de leurs usages et de leur insertion dans les champs de la culture ou de l'éducation (Meunier \& Peraya, 2010) ; le musée est alors envisagé comme un média éducatif (Cohen-Azria, 2002a). Cette approche reprend au moins en partie le postulat d'Albert Parr qui, dès 1962, considérait le musée comme un mass média (Parr, 1962) : « [...] Parr conclut que ce rapport à l'espace et au temps confère à l'exposition la propriété de s'adresser à tous de façon individuelle. C'est pourquoi le musée, comme institution, est pour lui un mass média. » (Schiele, 1992.)

L'article de Jean Davallon « Le musée est-il vraiment un média ? » paru dans le numéro 2 de la revue Publics \& Musées (Davallon, 1992) propose dès lors de questionner l'exposition en tant que média. La fortune de cette problématique s'explique : 1) par l'explosion du nombre d'expositions médias temporaires portant sur des thématiques de plus en plus précises ou spécialisées réalisées par les musées ; 2) par les résultats des études de publics qui confirment que les visiteurs déclarent aller voir une exposition avant tout pour apprendre et se cultiver (Poli \& Gottesdiener, 2008).

38 Donnant ainsi la primeur à la pratique sociale (l'éducation comme motivation à venir au musée), les chercheurs s'intéressent tout autant aux modalités de production du média exposition - et aux dispositifs communicationnels qui la constituent (textes, images, outils numériques) - qu'aux modalités de réception que les visiteurs s'ingénient à construire et à inventer en fonction de leurs attentes, de leurs pratiques d'autres médias et de leurs goûts culturels ou technologiques (livre, cinéma, théâtre, Internet, jeux vidéo, etc.) (Château, 1998 ; Vigué-Camus, 1998).

Une des difficultés de l'exercice auquel nous nous livrons ici consiste à tenter de scinder, à l'intérieur d'un ensemble de discours produits par la recherche en muséologie, une partie d'entre eux caractérisés par la problématique « éducation et musée » (Davallon, 1999). Or, la tâche est quasiment illusoire puisque, dans la majorité des recherches publiées en France depuis les années quatre-vingt-dix, la prise en compte du désir d'apprendre témoigné par le visiteur étant placée au centre des questions et des formalisations de la dimension communicationnelle de la visite au musée, la pratique du musée et l'apprentissage (libre ou encadré, subordonné ou critique) sont perçus comme inhérents l'un à l'autre (Bigot et al, 2012). Les recherches sur le musée et l'exposition comme médias n'échappent pas à cette représentation imposée, à cette " mythologie » (au sens de Barthes, 1970) du désir d'apprendre en se rendant au musée, pourrait-on dire (Deloche, 2010). D'autant que les travaux récents à propos des institutions de stockage des savoirs et des contenus, comme les bibliothèques et les musées, évoquent de concert les répercussions des technologies numériques et de la société de l'écran sur nos manières d'accéder à la connaissance, d'apprendre, de partager et de créer (Caron, 2011). Cette 
problématique et la mouvance des recherches en cours sur la culture numérique et la société de la connaissance, recherches constitutionnellement interdisciplinaires, produiront selon nous à l'avenir les connaissances essentielles pour poser un regard critique, novateur et constructif sur les rapports que les citoyens vivent et vivront avec le musée.

Notons toutefois que dans une perspective résolument critique, centrée sur les médias technologiques dans l'exposition, certains travaux critiquent de manière parfois virulente le recours aux multimédias et aux ordinateurs insérés dans les expositions, sans motivation véritable autre que celle de la fascination escomptée des visiteurs pour la technologie informatique (Weltzi-Faichild \& Dubé, 1998).

\section{Les notions opératoires}

L'analyse méthodique du discours des travaux consacrés à " éducation et musée » aboutit à repérer un certain nombre de vocables et de syntagmes qui composent le système ouvert des principales notions opératoires de ces recherches.

\section{Des vocables qui parlent de musée et d'éducation}

42 Comme c'est souvent le cas en sciences humaines et sociales, les chercheurs exploitent la flexibilité de la langue en constituant des syntagmes notionnels majoritairement composés de substantifs et d'adjectifs. Les plus occurrents sont : visée éducative des musées, éducation extrascolaire, éducation scientifique au musée, etc. Certains syntagmes notionnels - ou notions opératoires - sont composés de substantifs reliés par des prépositions : musée vecteur d'éducation, partenariat musée-éducation, mission d'éducation du musée, etc.

43 Il est notable de constater que le sens des notions auxquelles les chercheurs attribuent un signifié conceptuel n'est pas encore fixé par un glossaire stable ni rattaché à un cadre théorique strict auxquels tous se référeraient (comme en linguistique ou en chimie par exemple).

$44 \quad$ S’il n'est pas aisé d'envisager un champ conceptuel homogène et pertinent, c'est aussi parce que le sens des vocables varie, ces dernières années, en fonction du contexte de référence théorique de chaque article, c'est-à-dire en fonction de la problématique de chaque recherche (question de recherche, point de vue, cadrage théorique et méthodologie). Ce manque de cadre conceptuel largement partagé ou communément admis pose problème évidemment lorsqu'il s'agit de tenter une synthèse diachronique des recherches à partir des deux mots-clés « éducation » et « musée ».

45 Cette relative liberté des chercheurs vis-à-vis de la langue et des vocabulaires spécialisés tient surtout au fait que les recherches en question sont presque systématiquement de nature pluri- ou interdisciplinaire. L'éducation au musée étant un champ de recherche enraciné dans les domaines de la pédagogie, de la psychologie, de la sociologie et de la communication (Bolmont, 2003), il en résulte, sur le plan paradigmatique, la création de vocables composites dont au moins un des termes est issu de la discipline initiale du chercheur. Le vocabulaire du domaine se caractérise donc par un dialogisme (ou échange permanent) lexicographique actif entre différents champs des sciences humaines et sociales et les termes propres au monde du musée.

46 Les vocables les plus fréquemment actualisés dans les discours primaires sur « musée » et « éducation » dans les corpus de publications répertoriés sont : la mission d'éducation du musée, la fonction d'éducation du musée, le musée comme vecteur d'éducation, l'objectif d'éducation du musée, la visée éducative des musées, l'éducation muséale, le partenariat musée/éducation, musée/partenariat scolaire, éducation continue et musée, éducation populaire et musée, éducation culturelle et musée, éducation formelle à l'école 
versus éducation non formelle au musée, éducation extrascolaire, périscolaire, éducation scientifique au musée, éducation artistique au musée, éducation citoyenne et musée, éducation interculturelle.

Dans leur usage courant, la signification des mots (école, éducation, formation, mission, etc.) qui composent ces syntagmes notionnels varie selon le type de discours et de situation de communication dans lequel ils sont employés (Turmel, 1997).

Dans les corpus d'articles que nous avons analysés, ces mots du langage courant se trouvent en situation de cooccurrence (de proximité syntaxique) avec des termes qui relèvent du champ sémantique de la muséologie. C'est en cela que ces termes méritent d'être considérés comme des notions en cours d'élaboration dans le champ de la recherche en muséologie (Desvallées \& Mairesse, 2011).

On peut alors classer les notions précédemment listées selon qu'elles sont attachées à telle institution, à telle représentation sociale, à tel lieu, à tel type de participants, à tel domaine de compétence, à telle dimension symbolique.

\section{Les références à la mission éducative du musée}

Depuis les années quatre-vingt-dix, certaines recherches mettent en discussion les définitions académiques du musée - comme celle de I'ıсом placée au début de cet article pour les faire évoluer ou pour en développer une approche critique, partant du postulat que le musée est une institution à laquelle la société confère des missions, des objectifs, des visées, dont la fonction d'éducation du grand public à des questions de sciences et société (Fortin-Debart, 2004). L'éducation s'entend alors comme l'élément-clé de toute tentative de démocratisation car elle permet aux citoyens l'acquisition des connaissances, mais aussi la formation à des compétences et à des apprentissages indispensables pour participer aux mutations de la société et aux instances de décisions démocratiques. Les recherches de ce type amènent d'autres questions, comme celle de la tarification de l'entrée au musée, celle de l'éloignement de certains territoires par rapport aux institutions muséales, celle de la démocratisation de la culture, de la présence des différents métiers au musée, du lien entre idéologie, politique et culture (Seron, 2006).

51 Ces travaux restent parfaitement d'actualité aujourd'hui, notamment avec le recours aux outils numériques interactifs (ou non) dans les expositions, que certains chercheurs dénoncent comme une intrusion du monde marchand dans une institution qui, jusqu'à peu, s'en démarquait (Maigret, 2005). Les chercheurs qui se situent dans ce type de démarche recourent aux notions suivantes : mission d'éducation du musée, musée comme vecteur d'éducation, objectif d'éducation du musée, visée éducative des musées.

\section{Les références à l'interaction comme situation d'éducation}

Des enseignants-chercheurs en IUFM ou des universitaires en sciences de l'éducation ont participé à construire un courant de recherche au centre duquel le musée est en interaction avec l'école (primaire, collège, lycée) dans le cadre d'un partenariat officialisé par les deux institutions (Romano, 2001). De nombreuses recherches-actions ont été et sont menées sous l'égide de ce type de collaboration, dont le maitre mot est " partenariat » (Girault \& Guichard, 1995). Celui-ci y est envisagé et défendu comme « une expérience sociale nouvelle au cours de laquelle la médiation culturelle et didactique entre les enseignants, les intervenants des musées et les élèves, contribue à l'émergence d'un espace de coéducation » (Buffet, 1999). Largement développées au Canada dans les années quatre-vingt, quatre-vingt-dix, ces recherches trouvent un écho favorable en France dans les années quatre-vingt-dix grâce à la présence des enseignants de l'Éducation nationale détachés, plutôt dans les musées de sciences (Allard \& Boucher, 1998). L’intérêt majeur de 
ce type de recherche est de voir comment, par une analyse réflexive des interactions entre école et musée, le cadre ou contrat posé au départ du partenariat induit des changements réciproques dans les manières de travailler chez les professionnels du musée autant que chez les professionnels de l'école (Pélissier, 1995). Les recherches mettent l'accent sur le fait que ces interactions école/musée contribuent à donner une place centrale à la dimension sensible de l'apprentissage ainsi qu'à la force d'impact de l'ambiance (espace, esthétique, décors, éclairage, odeurs, sons, etc.), tant sur les médiateurs (enseignants et médiateurs de musée) que sur les visiteurs (élèves et enseignants) (Drouguet \& Gob, 2003). Depuis les années deux mille, ces recherches ont tendance à se recentrer sur des questionnements au départ didactiques, que le chercheur complexifie en optant pour des approches comparatives entre plusieurs musées et plusieurs écoles (parfois de villes ou même de pays différents) comme terrains d'expérimentation et de réflexion (Bolmont, 2003). Cécile Fortin-Debart relève notamment, dans une approche comparative, qu'en Grande-Bretagne les leviers favorables aux projets école-musée réussis sur le thème de l'engagement citoyen sont : 1) la liberté et l'autonomie des chefs d'établissement ; 2) la volonté politique d'intégrer les populations locales aux questions d'aménagement ; 3) l'engagement d'une personne-clé ayant des responsabilités dans le domaine de l'éducation ; 4) les aides extérieures des Urban Studies Centers ; 5) le réel ancrage local (Fortin-Debart \& Girault, 2009). Cette position va également dans le sens de la défense d'une muséographie participative - qui donne au visiteur la possibilité de saisir les positions des différents acteurs en jeu - et comparée - qui propose toujours au moins deux points de vue sur une question (Selbach, 2005).

Les notions auxquelles se rattachent ces recherches sont notamment : partenariat musée/éducation, musée/partenariat scolaire.

\section{Les références à la classification des publics cibles}

Le courant de recherche en lien avec la nouvelle muséologie, les écomusées et plus récemment la muséographie collaborative ou participative, met le citoyen au centre de ses problématiques et de ses discours. Ainsi, depuis plus de vingt ans (Desvallées et al. (dir), 1992), la littérature de recherche en muséologie foisonne-t-elle de travaux développant une vision muséale de la démocratisation culturelle. On pourrait dire que l'éducation sous toutes ses facettes (savoir, émotion, échange, esprit critique, innovation, création) est tellement au cœur des préoccupations des chercheurs qu'il ne s'agit plus de la circonscrire aux institutions (école, entreprise, musée, formation continue, théâtre, bibliothèque, etc.) mais de chercher à analyser comment chaque citoyen (dès l'âge de la scolarité) qui visite une exposition ou un musée ne sera plus la même personne après sa visite (Ruddel, 2005).

55 Les chercheurs qui s'inscrivent dans cette approche se réfèrent généralement à des conceptions philosophiques ou politiques de l'éducation ; les idées phares de l'éducation populaire reviennent en force actuellement, sans doute impulsées par les restrictions budgétaires imposées aux musées et par la crise économique qui touche les plus démunis dans leur rapport à la culture. Les notions d'« habitus », de " reproduction sociale », de « capital culturel » (Bourdieu \& Darbel, 1969) sont fréquemment revisitées par des chercheurs qui voient toutefois dans le musée (et par la gratuité d'accès pour les jeunes et les démunis) un média dont les potentialités d'éducation n'ont pas encore été vraiment exploitées (Meunier, Romano \& Jacobi, 2000 ; Eidelman, 2010).

L'éducation est ici envisagée exclusivement dans sa dimension non formelle, c'est-à-dire sans évaluation scolaire, sans diplôme, sans contrainte d'apprentissage (Schiele \& Jacobi, 1990).

57 On peut trouver quelques points communs entre ce type de recherche et les travaux actuels sur " Internet et éducation ", même si la comparaison entre les deux n'est pas encore traitée comme un objet de recherche à part entière (Vidal, 2003). 
$58 \quad$ L'intérêt majeur des recherches questionnant dans cette optique politique le rapport entre musée et éducation tient au fait qu'elles mobilisent des notions centrales dans les recherches actuelles sur la culture à l'ère du numérique, comme lecture/écriture, territoire, parité, démocratie participative, propriété intellectuelle, citoyenneté, culture (Poli, 2012).

59 Nous avons noté que ces recherches exploitent particulièrement les notions suivantes : éducation extrascolaire et musée, éducation périscolaire et musée, éducation formelle à l'école versus éducation non formelle au musée, éducation continue et musée, éducation populaire et musée, éducation culturelle et musée, éducation citoyenne et musée, éducation interculturelle et musée.

\section{Les références à la typologie des musées}

60 Reprenant à leur compte la distinction établie par le système scolaire entre les disciplines scientifiques et artistiques, certains chercheurs cloisonnent les expériences éducatives du musée en se fondant sur cette partition. Il ne faut pas s'en étonner puisque, pour des motifs liés aux collections et dès leurs origines, les musées sont historiquement répartis entre musées d'art, de sciences, d'histoire et, plus récemment, musées de société (Poulot, 2005). Les notions qui signalent ce type de recherches sont " éducation scientifique au musée » et « éducation artistique au musée ».

Pour autant, lorsque la recherche porte avant tout sur la médiation muséale et sur les registres de signes (langue, images, textes, mise en vitrine, codes sémiotiques, etc.), l'objet des analyses se focalise davantage sur les modalités du fonctionnement sémiotique de ces registres que sur les contenus disciplinaires médiatisés par l'exposition (Triquet, 2012). Ces recherches sur les outils et les dispositifs de médiation ont permis ces vingt dernières années de mettre en lumière certains fonctionnements communicationnels qui sont certes propres au musée, mais qui ne sont spécifiques ni à certains genres de connaissances particulières ni à certains types de muséographie. Dans des travaux récents sur la muséographie d'immersion (Belaën, 2004), dans laquelle l'ensemble des dispositifs de l'exposition est destiné à faire en sorte que le visiteur se sente immergé dans une ambiance, plus que mis en relation avec des savoirs, la distinction entre éducation scientifique et éducation artistique tend à s'effacer au profit d'une approche polysensorielle de l'expérience de rencontre avec les savoirs muséographiés. Il n'empêche que les recherches sur " éducation et musée ", à l'instar des études de réception auprès des publics enfants ou adultes, reconduisent toujours fréquemment la partition entre éducation scientifique et éducation artistique (Cordier, 2005). Les musées de beaux-arts et les centres d'art contemporain notamment - arguent des nouvelles mesures prises par le ministère de l'Éducation nationale à propos de l'éducation artistique et culturelle pour renforcer la spécialisation de leur discours sur histoire de l'art et esthétique (Davallon \& Le Marec, 2000). Certaines recherches se font l'écho d'expériences interdisciplinaires, comme lorsque le musée de société demande à un artiste contemporain de travailler in situ dans une exposition thématique, ou lorsqu'un centre de culture scientifique et technique invite les arts vivants à créer des spectacles qui, avec le langage de la danse, du théâtre ou des arts plastiques, parlent de science et de technologie (Bordeaux, 2009). Avec l'arrivée en force du numérique au musée (bornes in situ, connexions Internet, applications pour tablettes et téléphones intelligents), cette problématique de la médiation, non centrée sur tel ou tel domaine de la connaissance, ouvre de nouvelles perspectives de recherche sur « éducation et musée », décentrant la question des types de savoirs transmis sur la question des écritures numériques des savoirs, médiatisées par les écrits d'écran (Tardy \& Jeanneret, 2007). Il n'en reste pas moins que les formations discursives qui établissent la partition entre art, science et société restent prégnantes et pertinentes pour la grande majorité des discours éducatifs au sens large. Les enseignants 
en formation et en activité restent, on le constate, cloisonnés par ces balisages institutionnels venus autant du musée que de l'école (Triquet, 2001).

\section{Les références à l'éducation muséale}

La dernière notion de ce panorama lexicologique est celle d' « éducation muséale ». Nous avons choisi de la traiter après les autres, parce que nous la considérons comme centrale et clairement mobilisée depuis les années trente dans toute recherche sur l'éducation et le musée (Dufresne-Tassé, 1991). C'est son caractère récurrent et structurant dans ce champ de recherche qui permet de saisir la signification que l'on peut aujourd'hui attribuer à cette notion : 1) éduquer le public (réel et potentiel, adulte et enfant) au fonctionnement et aux enjeux du musée dans la société ; 2) offrir la possibilité à chacun de se saisir de ce que le musée peut lui apporter pour échanger, apprendre, critiquer, participer, s'exprimer, créer, enrichir sa personnalité (Lussier-Desrochers, Lemerise \& Vitor, 2002).

Partant de cette notion, l'essentiel est de donner les clés de compréhension du musée comme dispositif éducatif global. Dans cette conception de l'éducation, le musée doit s'ouvrir à la pluralité des discours et se donner la chance d'être un lieu de débat, d'expression démocratique (Rasse, 1999).

64 Les médiateurs au musée ont alors pour mission de faire découvrir par l'apprentissage et par l'émotion comment utiliser les expositions pour apprendre ou se délecter (et non seulement apprendre d'une exposition), pourquoi on construit et on enrichit une collection, les différents métiers qui participent à la conception d'une exposition (et non pas écouter un médiateur faire un exposé adapté à l'espace muséal), comment on gère les coulisses du musée (accueil des visiteurs, sécurité, entretien).

65 On pourrait dire que la notion d'éducation muséale revient à donner du sens, de manière concrète et vivante, à chacun des énoncés et donc à chacune des fonctions de la définition du musée proposée par I'ıсом et citée plus haut.

66 C'est avant tout cette dimension réflexive à laquelle invite la notion d'éducation muséale que nous retiendrons ici comme la plus innovante pour la recherche en muséologie. Nous y trouvons l'ambition culturelle et la dimension critique qui manquent fréquemment aux recherches centrées soit sur des contenus, soit sur des apprentissages disciplinaires.

\section{Conclusion}

L'article de Duncan Cameron « Un point de vue, le musée considéré comme système de communication et les implications de ce système dans les programmes éducatifs muséaux », fréquemment cité depuis sa publication en 1992 dans Vagues : une anthologie de la nouvelle muséologie, fait figure de référence emblématique pour tout un courant de recherches françaises qui, depuis une vingtaine d'années, questionne le musée en tant que système de communication dans sa présumée consubstantielle vocation éducative et communicationnelle (Cameron, [1968] 1992).

Depuis, d'autres auteurs, à l'instar de Françoise Buffet, Élisabeth Caillet, Jean Davallon, André Desvallées, Jacqueline Eidelman, Hana Gottesdiener, Daniel Guichard, Yves Girault, Daniel Jacobi, Michel Van Praët - pour ne citer que ceux qui ont publié et encadré des recherches -, ont élaboré le socle épistémologique et méthodologique des travaux sur " apprendre au et par le musée ».

69 Éducation populaire, formation à l'esprit critique des publics adultes, expression citoyenne au musée, impact des études de publics sur les médiations de savoirs et sur les dialogues avec les publics : ces questions à dimension éducative sont centrales dans les recherches des années quatre-vingt-dix à deux mille dix menées par Serge Chaumier, Joëlle Le Marec, Marie-Sylvie Poli, Paul Rasse ou Eric Triquet, et par de jeunes chercheurs comme Anne Jonchery. 
70 Il est également intéressant de constater que dans les formations qu'il organise pour les professionnels du champ muséal, l'office de Coopération et d'information muséales (Ocim) propose régulièrement des sessions animées par des chercheurs sur « éducation et musée ».

71 Parmi les recherches conduites en partenariat avec des professionnels de musée, les démarches de conception d'expositions collaboratives et participatives émergent comme un champ récent, propice à des travaux exploratoires qui tentent de formaliser la place et le rôle du public lorsque celui-ci prend la parole, participant ainsi aux différents espaces de production, de socialisation et de médiation du musée (Duclos, 2011).

72 La prise en compte récente de l'urgence, pour les institutions comme le musée, à faire de la société du numérique un objet de recherche interdisciplinaire, relance aujourd'hui avec profit les débats sur l'éducation et le musée (Doueihi, 2011 ; Kambouchner, Meirieu \& Stiegler, 2012).

73 À l'issue de ce tour d'horizon de la recherche menée en France sur " éducation et musée » depuis la parution en 1992 du numéro 1 de Publics \& Musées, il est évident qu'un vaste programme de recherches est devant nous. Ce programme, dont on conçoit bien qu'il sera interdisciplinaire, est déjà impulsé en partie par les transformations que le numérique introduit de gré ou de force dans les relations entre l'humain et le savoir, avec l'offensive du marketing culturel, rendant de plus en plus difficile l'élaboration du sens critique.

74 Or, le musée, parce qu'il continue de permettre de croiser physiquement et intellectuellement d'autres que soi, venus au même moment, dans ce même lieu, avec la même envie de partager une compréhension du monde, permet précisément de repenser aujourd'hui, à l'ère de l'écran et du numérique tout puissants, la question du plaisir à partager de l'intelligence et du sensible, du savoir et de la découverte.

75 Il n'est pas, pour en être convaincu, indispensable de se référer aux chiffres de fréquentation des expositions temporaires à succès. Observer des visiteurs qui regardent des œuvres ou des objets, lisent des cartels ou des écrans, flânent, communiquent entre eux, suffit amplement à susciter l'envie d'entreprendre de nouveaux chantiers de recherche sur l'éducation et le musée pour les vingt années à venir.

\section{Bibliographie}

Ailincai (Rodica). 2008. " Un dispositif de sensibilisation des parents à leur rôle éducatif auprès de leurs enfants dans un musée scientifique », p. 291-298 in Précarités et Éducation familiale, sous la direction de Chantal Zaouche Gaudron. Paris : Éd. Eres.

Allard (Michel) \& Boucher (Suzanne). 1998. Éduquer au musée : un modèle théorique de pédagogie muséale. Montréal : Éd. Hurtubise / HMH.

Barthes (Roland). 1970. Mythologies. Paris : Éd. du Seuil.

Belaën (Florence). 2004. "L'immersion d'un nouveau mode de médiation au musée des Sciences. Étude de cas : la présentation du changement climatique ", p. 91-110 in Actes du colloque Sciences médias et société, sous la direction de Joëlle Le Marec \& Igor Babou. ENS Lyon, 15-17 juin 2004. [En ligne] <http://sciences-medias.ens-lyon.fr/article>

Bigot (Régis) et al. 2012. "La visite des musées, des expositions et des monuments ». Collection des rapports du CREDOC, 281. [En ligne] <http://www.credoc.fr/pdf/Rapp/281.pdf>

Bolmont (Étienne). 2003. "Lieux de découverte : Les musées pour enseigner la science et la technologie ", p. 61-70 in Enseigner la science et la technologie, sous la direction de Maria Xanthoudaki. [En ligne] <http://chercheur.me/-a+place+discover+museum+resource+education>

Bordeaux (Marie-Christine). 2009. «L'éducation artistique : Un partenariat inachevé », p. 419-455 in Une ambition partagée ? La coopération entre le ministère de la Culture et les collectivités territoriales (1959-2009), sous la direction de Philippe Poirier \& René Rizzardo. Paris : Éd. La documentation Française.

Bourdieu (Pierre) \& Darbel (Alain). 1969. L’Amour de l'art : Les musées et leur public. Paris : Éd. de Minuit.

Boyer (Myriam). 2009. Les Collections et Les Muséographies des musées de l'école et de l'éducation en Europe : Étude comparative. Thèse de doctorat : Épistémologie, histoire des sciences et 
techniques : Conservatoire national des arts et métiers.

Buffet (Françoise). 1999. Entre école et musée : Le partenariat culturel d'éducation. Lyon : Presses universitaires de Lyon.

Cameron (Duncan). [1968] 1992. "Un point de vue : le musée considéré comme système de communication et les implications de ce système dans les programmes éducatifs muséaux », p. 259288 in Vagues : Une anthologie de la nouvelle muséologie, vol. 1, sous la direction d'André Desvallées (et. al.). Mâcon / Savigny-le-Temple : Éd. W / MNES.

Caron (Daniel). 2011. Web HT.o Pour une société informée : La pertinence du numérique et ses défis pour les sociétés démocratiques du XXIe siècle. Paris : Éd. Hermann.

Chaléon (Armelle), Demarq (Catherine) \& Maggioni (Ludovic). 2010. « Adapter une exposition pour le jeune public ». La Lettre de l'Ocim, 132, p. 21-27.

Château (Dominique). 1998. « Conscience esthétique et expertise : La collaboration entre Dewey et Barnes ». Publics \& Musées, 14, p. 39-53.

DOI : 10.3406/pumus.1998.1115

Chaumier (Serge). 2008. « Le public, acteur de la production d'exposition ? Un modèle écartelé entre enthousiasme et réticence ", p. 241-250 in La Place des publics : De l'étude des usages et recherches par les musées, sous la direction de Jacqueline Eidelman, Mélanie Roustan \& Bernadette Goldstein. Paris : Éd. La documentation Française.

Chauvey (Valérie). 2010. «Le texte au musée pour les visiteurs non-voyants ». La Lettre de l'Ocim, 132, p. 4-47.

Cohen-Azria (Cora). 2000. Contribution à l'étude des relations entre l'école et le musée. Thèse de doctorat : Muséologie des sciences de la nature et de l'homme : Muséum national d'histoire naturelle.

Cohen-Azria (Cora). 2002a. Quand l'enfant devient visiteur : Nouvelle approche du partenariat école / musée. Paris : Éd. de l'Harmattan.

Cohen-Azria (Cora). 2002b. « L'enfant, l'élève et le visiteur ». La Lettre de l'Ocim, 8o, p. 32-37.

Colardelle (Michel). 2007. « La mission sociale des musées et son illustration au cœur du projet scientifique et culturel du futur musée des civilisations de l'Europe et de la Méditérranée » in Actes du colloque : La collaboration entre les musées de France et les fédérations d'éducation populaire. Angers-Château d’Écouen, mars 2006. Paris : Éd. La documentation Française.

Cordier (Jean-Pierre) \& Serre (Sylvie). 2000. "Interactions familiales au musée : Approches sociologiques et cognitives ", p. 11-22 in La Muséologie des sciences et ses publics : Regards croisés sur la grande galerie de l'évolution du Museum d'histoire naturelle, sous la direction de Jacqueline Eidelman \& Michel Van Praët. Paris : Presses universitaires de France.

Cordier (Jean-Pierre). 2005. « La reconnaissance de soi et ses limites ». Culture \& Musées, 6, p. 4363.

Davallon (Jean). 1992. « Le musée est-il vraiment un média ? ». Publics \& Musées, 2, p. 99-123.

DOI : 10.3406/pumus.1992.1017

Davallon (Jean). 1999. L'Exposition à l'œuvre. Paris : Éd. de l'Harmattan.

Davallon (Jean) \& Le Marec (Joëlle). 2000. «L'usage en son contexte sur les usages des interactifs et des cédéroms des musées ». Réseaux, 101, vol. 18, p. 173-196.

Deloche (Bernard). (2010). Mythologie du musée. Paris : Éd. Le cavalier bleu.

Desvallées (André) et. al. (dir.). 1992. Vagues : Une anthologie de la nouvelle muséologie, vol. 1. Mâcon / Savigny-le-Temple : Éd. W / MNES.

Desvallées (André) \& Mairesse (François). 2005. "Sur la muséologie ». Culture \& Musées, 6, p. 131155 .

Desvallées (André) \& Mairesse (François). 2011. Dictionnaire de muséologie. Paris : Armand Colin.

Dierking (Lynn D.). 1994. « Rôle de l'interaction sociale dans l'expérience muséale ». Publics \& Musées, 5, p. 19-43.

Doueihi (Milad). 2011. Pour un humanisme numérique. Paris : Éd. du Seuil.

Drouguet (Noémie) \& Gob (André). 2003. " La conception d’une exposition : Du schéma programmatique à la mise en espace ». Culture \& Musées, 2, p. 147-157.

Duclos (Jean-Claude). 2011. "La coécriture au musée », p. 111-118 in La Fabrique du musée de sciences et sociétés, sous la direction de Michel Côté. Paris : Éd. La Documentation française.

Dufresne-Tassé (Colette). 1991. « L'éducation muséale, son rôle, sa spécificité, sa place parmi les autres fonctions du musée ». Revue canadienne de l’éducation, 3, vol. 16, p. 251-257. 
Eidelman (Jacqueline), Roustan (Mélanie) \& Goldstein (Bernadette) (dir.). 2007. La Place des publics : De l'usage des études et recherches par les musées. Paris : Éd. La documentation Française.

Eidelman (Jacqueline). 2010. « Du nonpublic des musées aux publics de la gratuité ». Loisirs et société, 32-1, p. 173-200.

Eidelman (Jacqueline) \& Jonchery (Anne). 2011. "Sociologie de la démocratisation des musées ». Hermès, 61, p. 52-6o.

Flon (Émilie). 2006. « L'exposition d'archéologie et le phénomène de spectacularisation ». Musées \& Collections publiques de France, 247, p. 32-37.

Fortin-Debart (Cécile). 2004. Le Partenariat école-musée pour une éducation à l'environnement. Paris : Éd. de l'Harmattan. (Savoir et formation.)

Fortin-Debart (Cécile) \& Girault (Yves). 2009. « De l'analyse des pratiques de participation citoyenne à des propositions pour une éducation relative à l'environnement ». Éducation relative à l'environnement, 8, p. 129-145.

Girault (Yves) \& Guichard (Jacques). 1995. « Problématique et enjeux du partenariat école / musée à la grande galerie de l'évolution ». Publics \& Musées, 7, p. 69-91.

Girault (Yves) (dir). 2003. L’Accueil du public scolaire dans les muséums. Paris : Éd. de l'Harmattan.

Gottesdiener (Hana). 1992. Freins et Motivations à la visite des musées d'art. Paris : Éd. Département des études, de la prospective et des statistiques / Ministère de la Culture.

Jonchery (Anne). 2005. Quand la famille vient au musée. Thèse de doctorat : Muséologie des sciences naturelles et humaines : Muséum national d'histoire naturelle.

Julien (Louise). 2004. « Passeurs de culture ». Montréal Cultures, 5, p. 6-18.

Kambouchner (Denis), Meirieu (Philippe) \& Stiegler (Bernard). 2012. L’École, le Numérique et la Société qui vient. Paris : Éd. Mille et une nuits.

Kindler (Anna M.) \& Darras (Bernard). 1998. « Le musée, l'école et l'éducation artistique ». Publics \& musées, 14, p. 15-37.

Le Marec (Joëlle). 2006. "Les musées et bibliothèques comme espaces culturels de formation ». Savoirs, 11, p. 9-38.

Lussier-Desrochers (Dany), Lemerise (Tamara) \& Vitor (Matias). 2002. Courants contemporains de la recherche en éducation muséale. Québec : Éd. MultiMonde.

Maigret (Jacques). 2005. " Éducation, tourisme, économie : l’impossible pari des parcs scientifiques ». Culture \& Musées, 5, p. 168-178.

Mairesse (François). 2000. « La belle histoire aux origines de la nouvelle muséologie ». Publics \& Musées, 17-18, p. 33-56.

Mariani-Ducray (Francine) \& Wasserman (Françoise). 2007. Actes du colloque : La collaboration entre les musées de France et les fédérations d'éducation populaire. AngersChâteau d'Écouen, mars 2006. Paris : Éd. La Documentation française.

Maurel (Christian). 2009. "Éducation populaire : Disparition ou mutation ? ». Vie sociale, 4, p. 4-5.

Meunier (Anik), Romano (Sylvie) \& Jacobi (Daniel). 200o. « La médiation culturelle dans les musées : Une forme de régulation sociale ». Recherches en communication, 13, p. 37-60.

Meunier (Anik). 2008. " L'éducation muséale, un rapport au savoir ». Recherches en communication, 29, p. 101-124

Meunier (Jean-Pierre) \& Peraya (Daniel). 2010. Introduction aux théories de la communication. Bruxelles : De Boeck.

Molinati (Grégoire) \& Girault (Yves). 2007. « La médiation muséale des neurosciences : Quatre expositions récentes sur le cerveau ». Culture \& Musées, 10, p. 97-123.

DOI : 10.3406/pumus.2007.1442

Parr (Albert E.). 1962. « Patterns of Progress in Exhibition ». Curator, 5, p. 29-345.

Pélissier (Gilbert). 1995. «L'école et le musée : Croisement des savoirs ? » Actes du colloque : L'école et le musée. Lille: Palais des beaux-arts.

Poli (Marie-Sylvie). 2002. Le Texte au musée : Une approche sémiotique. Paris : Éd. de l'Harmattan.

Poli (Marie-Sylvie). (2008). " L'effet didactique des textes poétiques dans une exposition de peinture ». Synergies, 1, p. 163-172.

Poli (Marie-Sylvie) \& Gottesdiener (Hana). 2008. « Les titres d'exposition : sur quoi communiquent les musées ». Culture \& Musées, 11, p. 81-88. 
Poli (Marie-Sylvie) \& Idjeraoui-Ravez (Linda). 2011. "Représentations de l'immigration dans les musées d'histoire ». Hermès, 61, p. 138-144.

Poli (Marie-Sylvie). 2012. "Nanotechnologies : Questionner la convergence culture scientifique / opinion publique. Le cas de l'exposition Nanotechnologies infiniment petit maxi défis ! », p. 285-301 in Les Masques de la convergence. Enquêtes sur sciences industries et aménagements, sous la direction de Bernard Miège \& Dominique Vinck. Paris : Éd. des Archives contemporaines.

Porcedda (Aude). 2006. Les Défis du changement. Thèse de doctorat : Muséologie des sciences naturelles et humaines, muséologie, sociologie : Muséum national d'histoire naturelle.

Poulot (Dominique). 2005. Une histoire des musées de France, XVIIIe-XXe siècles. Paris : Éd. La Découverte. (L'espace de l'Histoire.)

Profit (Aline). 2009. Le Musée de la maison Hébert. Mémoire de recherche : Lettres et arts : Université StendhalGrenoble.

Publics \& Musées, 7, 1995. Musées et éducation, sous la direction de Daniel Jacobi \& Odile Coppey.

Rasse (Paul). 1999. Les Musées à la lumière de l'espace public : Histoire, évolution, enjeux. Paris : Éd. de l'Harmattan.

Rigat (Françoise). 2005. « Les textes expographiques : Pour une approche de la langue-culture dans les expositions d'art moderne ». Ela, 138, p. 153-170.

DOI : 10.3917/ela.138.0153

Romano (Sylvie). 2001. "Les enfants de 4 à 6 ans et les institutions culturelles ». Recherches en communication, 5, p. 4-21.

Ruddel (Thierry). 2005. "Musées civilisants du Québec et du Canada : Les enjeux politiques et publics ». Culture \& musées, 6, p. 156-165.

Schiele (Bernard) \& Jacobi (Daniel). 1990. " La vulgarisation scientifique et l'éducation non formelle ». Revue française depédagogie, 91, p. 81-111.

Schiele (Bernard). 1992. "L'invention simultanée du visiteur et de l'exposition ». Publics \& musées, 2, p. 71-98.

Schiele (Bernard) \& Boucher (Louise). 2001. " Note sur la médiation muséale contemporaine ». Quaderni, 46, p. 27-51.

Selbach (Gérard). 2005. « Publics et muséologie amérindienne ». Culture \& musées, 6, p. 21-42.

Seron (Emmanuelle). (2006). La Muséologie participative : concepts et expérimentations. L'expérience d'un comité de visiteur au nouveau musée de l'Homme. Mémoire de Master 2 Recherche : Culture et communication (section muséologie) : Université d'Avignon et des Pays de Vaucluse.

Tardy (Cécile) \& Jeanneret (Yves) (sous la direction de). 2007. L'Écriture des médias informatisés : Espaces de pratiques. Paris : Éd. Hermès Sciences-Lavoisier. (Systèmes d'information et organisations documentaires.)

Topalian (Roland) \& Le Marec (Joëlle). 2003. «Énonciation plurielle et publication de la parole du public en contexte muséal : Le cas de la tribune des visiteurs ». Communication et langages, 135, p. 12-24.

Triquet (Éric). 2001. «La visite scolaire au musée : regards croisés d'enseignants en formation initiale et en activité ». Skhole, $\mathrm{n}^{\circ}$ hors-série, p. 19-38.

Triquet (Éric) \& Poli (Marie-Sylvie). 2001. «Écrire au muséum : Pour une approche résolument transdisciplinaire ». Lidil, 23, p. 45-68.

Triquet (Éric). 2012. « Le récit dans la médiation des sciences et techniques. Introduction ». Culture \& musées, 18, p. 8-15.

Turmel (André). 1997. "Le retour du concept d'institution », p. 1-24 in Culture, Institution et Savoir : Culture française d'Amérique. Québec : Presses universitaires de Laval.

Vidal (Geneviève). 2003. "Interactivité et médiation dans l'usage des multimédias de musées ». Communication et langages, 137, p. 63-76.

Vigué-Camus (Agnès). 1998. «Une approche des usages et représentations des écrans multimédias dans leur contexte social de production ». Publics \& Musées, 13, p. 43-65.

Weltzi-Faichild (Andréa) \& Dubé (Louis). 1998. « Le multimédia peut-il aider à réduire la dissonance cognitive ? ». Publics \& musées, 13, p. 17-28.

\section{Pour citer cet article}


Référence papier

Marie-Sylvie Poli, « Éducation et musée », Culture \& Musées, Hors-série | 2013, 165-187.

Référence électronique

Marie-Sylvie Poli, «Éducation et musée », Culture \& Musées [En ligne], Hors-série | 2013, mis en ligne le 19 juin 2018, consulté le 25 juin 2021. URL :

http://journals.openedition.org/culturemusees/757 ; DOI :

https://doi.org/10.4000/culturemusees.757

\section{Auteur}

\section{Marie-Sylvie Poli}

Marie-Sylvie Poli est professeur à l'université d'Avignon et des Pays de Vaucluse en sciences de l'information et de la communication, membre du centre Norbert Elias (UMR CNRS 8562), équipe «Culture et communication ». Elle est codirectrice de la structure fédérative de recherche Agorantic «Culture, Patrimoines, Sociétés numériques » (UAPVICNRS). Ses travaux portent sur la sémiologie de la médiation des savoirs dans le champ muséal et sur les nouvelles formes de la critique, notamment sur Internet.

marie-sylvie.poli@univ-avignon.fr

Articles du même auteur

La parade numérique du Musée du Désert [Texte intégral]

Paru dans Culture \& Musées, 37 | 2021

Hommage à Marie-Claire Habib [Texte intégral]

Paru dans Culture \& Musées, 36 | 2020

Visite du Musée Ghibli, à Tokyo [Texte intégral]

Paru dans Culture \& Musées, 36 | 2020

Artistes \& Robots [Texte intégral]

Grand Palais, Galeries nationales, Paris, 5 avril - 9 juillet 2018

Paru dans Culture \& Musées, 35 | 2020

Marie-Pierre Fourquet-Courbet \& Didier Courbet. Connectés et heureux ! Du stress digital au bien-être numérique [Texte intégral]

Paru dans Culture \& Musées, 35 | 2020

Cités millénaires. Voyage virtuel de Palmyre à Mossoul [Texte intégral] Institut du monde arabe (IMA), Paris, 10 octobre 2018 - 17 février 2019

Paru dans Culture \& Musées, 35 | 2020

Tous les textes...

\section{Droits d'auteur}

Culture \& Musées 